\title{
Special Genotypes of ABA Key Functional Genes NCEDs Members in the Glycyrrhizic Acid Synthesis Regulatory Network Promote Glycyrrhizic Acid Synthesis in Licorice
}

Ting Li ( $\sim$ Abby1230@163.com )

Beijing University of Chinese Medicine

Feiyang Xuan

Beijing University of Chinese Medicine

Xiaoying Shen

Beijing University of Chinese Medicine

Dan Jiang

Beijing University of Chinese Medicine

Guangxi Ren

Beijing University of Chinese Medicine

Chunsheng Liu

Beijing University of Chinese Medicine

\section{Research}

Keywords: Abscisic acid, Glycyrrhizic acid, Licorice, NCEDs, Network

Posted Date: October 12th, 2021

DOI: https://doi.org/10.21203/rs.3.rs-915680/v1

License: (c) (1) This work is licensed under a Creative Commons Attribution 4.0 International License.

Read Full License 


\section{Abstract}

\section{Background}

The glycyrrhizic acid biosynthesis pathway does not exist in isolation, but is connected with the biosynthesis pathways of other secondary metabolites in licorice and finally forms a network. Our previous study found that exogenous spraying of appropriate concentration of abscisic acid (ABA) could increase the content of glycyrrhizic acid (GA) in licorice. However, the mechanism of action remains unknown. We aim to understand the molecular mechanism of ABA promoting the synthesis of GA in licorice and find the molecular marker for the high content of GA germplasm material.

Methods

We analyzed the expression of the key gene of $\beta-A S$ for GA synthesis after applying ABA, the key functional genes NCED1, NCED3 and NCED4 in the process of ABA synthesis were overexpression, and analyzed the relationship between the SNP polymorphism of the NCED1, NCED3, NCED4 and the content of the GA and $A B A$ in 13 different provenances of licorice with the grey correlation analysis.

Results

The appropriate concentration of ABA treatment could increase the content of the GA through improving the expression of $\beta-A S$. There were significant differences in the content of ABA and GA among the 13 provenances, and the 3 members of the NCEDs family of different provenances had abundant SNP variation sites. Grey correlation and overexpression of NCEDs function both showed that the effect of promoting the synthesis of ABA and GA: NCED1 437 bp G type > NCED3 966 bp G type > NCED4 845 bp A type. All of the above indicated that NCEDs gene variation was the reason for the diversity of GA and ABA content. When selecting high GA germplasm, more priority should be given to NCED1 gene 437 bp G type, NCED3 gene 966 bp G type and NCED4 gene 845 bp A type.

\section{Conclusion}

This study provides a basis for the selection of excellent GA content germplasm of licorice, and provide some reference for producing a high quality cultivated licorice.

\section{Introduction}

Licorice is the most important bulk medicinal material, and it is also an important additive in cosmetics, health care products, tobacco and other industries. The annual demand is huge [1]. Modern pharmacology has proved that the licorice and its extract has anti-inflammatory and antiviral antiulcer, antiinflammation, spasmolysis, antioxidative, contravariance, antiviral, anticancer activities, hepatoprotective, eliminating phlegm and reinforcing remembrance effects [2]. Triterpene saponins are the main chemical components of licorice [3] , which mainly include glycyrrhizic acid (GA) and glycyrrhetinic acid. GA is more popular than glycyrrhetinic acid, which can exhibit potent biological 
activity including antibacterial, antiproliferative, anti-HINI anti-cancer and anti-HIV properties [2][4-6]. Besides, GA can protect plants from insects and microorganisms, and improve their resistance under harsh environmental conditions [3]. Before the 1980s, commercial licorice was derived from wild resources. In recent years, due to overexploitation, wild resources have become increasingly scarce. Cultivated licorice has become the main source of mainstream commodities [7] . However, most cultivated licorice has problems such as low quality, especially the content of GA is quite different from that of the wild licorice, which seriously affects the clinical efficacy of licorice and the development of its application. Therefore, it is of great significance to improve the content of GA and produce the high quality cultivated licorice in the research industry and production fields [8-9]. At present, some researchers are devoted to increasing the content of GA in cultivated licorice, such as planting in local areas, improving cultivation techniques [9][10], improving the cultivation environment [11][12] and extending planting years [13], etc. However, there are few studies on the genetic and molecular markers of licorice for the seed breeding. Thus, analyzing the genetic mechanism of GA content changes and breeding through molecular markers, excellent breeding quality is an effective measure to improve the quality of cultivated licorice.

Abscisic acid (ABA) is an important plant hormone, which plays an important role in regulating the growth and development of plants, adapting to the external environment, and the synthesis and accumulation of plant secondary metabolites [14][15][16][17]. At present, the research of related enzymes and genes in the ABA synthesis pathway is very clear. Phytoene synthase (PSY), zeaxanthin cyclase $(Z E P)$ and 9-cis-epoxycarotenoids $(N E C D)$ and aldehyde oxidase $(A O)$ are the main enzymes in the process of ABA synthesis [18]. Among them, NCED oxidizes 9-cis-epoxy carotenoids to C15 in the plastids and its release into the cytoplasm for downstream reactions are the key rate-limiting enzymes for $A B A$ synthesis [19][20][21]. There are polymorphisms in functional genes, and they have certain effects on the content of GA [22][23]. In addition, the synthetic pathways of plant secondary metabolites are complex and involve many enzymes. Therefore, the polymorphism of key enzymes and their coding genes is closely related to the biosynthesis of secondary metabolites.

At present, lots of literatures have shown that there is an interconnected and interrelated metabolic network in plant secondary metabolic synthesis pathways [8][24] . Each secondary metabolite pathway of licorice is not isolated. There are close connections between the various metabolic pathways. The common substrate is the node to form a network, and the different metabolic pathways within the network inyeract with each other[8]. In previous study, we applied different concentrations of Abscisic acid, Gibberellin, Methyl jasmonate, 6-Benzylaminopurine, Brassinolide and Auxin to licorice. After applying hormones to licorice, the seven components including GA, liquiritin apioside, Isoliquiritigenin, Isoliquiritin apioside, Isoliquiritin, Liquiritin and liquiritigenin were determined, and the correlation analysis was carried out, and the schematic diagram of GA regulation and control network was obtained.

Meanwhile, we found that ABA could regulate the synthesis and accumulation of GA [25]. Therefore, does the promotion effect of $A B A$ on the synthesis of GA increase the expression efficiency of key functional genes in the synthesis pathway, thereby promoting the synthesis of GA? How about the polymorphism of 
NCEDs members among different germplasms of licorice? Can the polymorphism of NCEDs members promote the accumulation of $A B A$ and $G A$ ?

Based on this, the main objectives of this study are as follows: 1) The expression of functional genes for GA synthesis after ABA treatment. 2) Starting from the substitution network that promotes the synthesis of GA, the key genes of ABA endogenous regulatory substances NCEDs were cloned, analyzed, verified and treated with inducing factors, and the mechanism of promoting GA synthesis was analyzed. 3 ) Through the analysis of the variation of ABA content in different germplasms of licorice, the status of $A B A$ in different germplasms under natural state could be understand. Through decomposing the correlation between the polymorphism of the key functional genes of $A B A$ synthesis and the content of $A B A$ and $G A$, analyze the molecular mechanism of the variation of ABA key functions affecting the differential formation of GA could be analyzied. This study provided the basis for explaining the difference of GA synthesis efficiency of different provenances, and supported for the selection of licorice germplasm with high GA content.

\section{Material And Methods}

\subsection{Materials}

The seeds of different provenances was collected from 13 main producing areas of licorice in China (marked as YX,HT,AH,CF,JQ,HJ1,HJ2,ZZ,ETK,GD,YC,MQ,KEL). The information was shown in Table 1. They were sown in the Medicinal Botanical Garden of Beijing University of Chinese Medicine and harvested one and a half years later. The licorice used to extract RNA and DNA were sown in $15 \mathrm{~cm} \times$ $12 \mathrm{~cm}$ flowerpots (Sand: Vermiculite $=1: 1$ ) and cultivated in an artificial climate incubator with a temperature of $25 \pm 2{ }^{\circ} \mathrm{C}$ and a light time of $14 \mathrm{~h} / \mathrm{d}$.

2.2 The effect of ABA treatment on the expression of key functional gene $\beta$-AS in licorice

The licorice was treated with different concentrations of ABA after 60 days of growth from seeds, the concentrations were $25,50,100$ and $200 \mathrm{mg} / \mathrm{L}$ respectively, each treatment was repeated three times, and pure water was used as the control group. Samples were taken at 3, 6, 12 and 24 hours of treatment, 90 $\mathrm{mg}$ root samples were frozen in liquid nitrogen and stored at $-80^{\circ} \mathrm{C}$. Then RNA was extracted according to RNA Extraction Kit (Biomed, Beijing). After extraction, reverse transcription was performed. Reverse transcription reaction system: $10 \mu \mathrm{L}$ of the last reaction solution, $1 \mu \mathrm{L}$ of primer script RT enzyme mix, 1 $\mu \mathrm{L}$ of RT primer mix, $4 \mu \mathrm{L}$ of $5 \times$ primer script buffer, $4 \mu \mathrm{L}$ of RNase free water, the total system was $10 \mu \mathrm{L}$ (The primer sequences were in Supplementary Materials Table 2). The conditions of reverse transcription were $37{ }^{\circ} \mathrm{C}, 15 \mathrm{~min}, 85^{\circ} \mathrm{C} 5 \mathrm{~s}$ and $4{ }^{\circ} \mathrm{C}$. Real time PCR reaction system: SYBR premix ex Taq $10 \mu \mathrm{L}, \mathrm{F}$ primer $0.4 \mu \mathrm{L}$, R primer $0.4 \mu \mathrm{L}$, cDNA template $2 \mu \mathrm{L}$, dd $\mathrm{d}_{2} \mathrm{O} 7.2 \mu \mathrm{L}$, total system $20 \mu \mathrm{L}$. Reaction conditions: pre denaturation at $95^{\circ} \mathrm{C}$ for $3 \mathrm{~min}$, denaturation at $95^{\circ} \mathrm{C}$ for $30 \mathrm{~s}$, annealing at $57^{\circ} \mathrm{C}$ for 30 $\mathrm{s}$, extension at $72{ }^{\circ} \mathrm{C}$ for $50 \mathrm{~s}, 40$ cycles.

2.3 Analysis of effective components and ABA variation of licorice from different provenances 
The licorice that grows for one and a half years was collected. The determination of ABA on the root tip of licorice was measured with ELISA kit, referring to the instruction manual for specific operation. The method for determining the effective components of licorice was as follows: $0.15 \mathrm{~g}$ of constant weight licorice powder was weighed, then it was putted into a $100 \mathrm{~mL}$ conical flask with stopper, $40 \mathrm{~mL} 70 \%$ ethanol was added and weighed it tightly. It was treated with ultrasound for $30 \mathrm{~min}$, maked up for the weight loss with $70 \%$ ethanol, shaked it well and passed through $0.45 \mu \mathrm{m}$ filter membrane for standby. An appropriate amount of GA reference substances were accurately weighed, and added with methanol to prepare a mixed reference solution with GA $110.80 \mu \mathrm{g} / \mathrm{mL}$. The chromatographic conditions were as follows: dual wavelength HPLC detection. Mobile phase: acetonitrile (A) $-0.05 \%$ phosphoric acid water (B); gradient elution: $0 \sim 8$ min, 20\% A; 8 30 min, 20\% 35\% A; $30 \sim 42$ min, 35\% 45\% A. Detection wavelength: $237 \mathrm{~nm}$ for $\mathrm{GA}$. The flow rate was $1.0 \mathrm{~mL} / \mathrm{min}$, the column temperature was $30{ }^{\circ} \mathrm{C}$, and the injection volume was $10 \mu \mathrm{L}$. Each sample was repeated three times.

\subsection{Cloning of NCEDs from licorice}

The whole plant of licorice growing for 30 days was used to extract the total RNA with the plant total RNA Extraction Kit (Biomed, Beijing), and then reverse transcription was performed to synthesize cDNA. Primers were designed according to the homologous regions of the reported NCED genes (NCED1, NCED3, NCED4), and 3 'and 5' RACE. The full-length primer sequences of the genes were shown in Supplementary Materials Table 3, Table 4, Table 5. The open reading frame of NCEDs was amplified. The primers were connected to pMD19T (pMD-NCED1, pMD-NCED3, pMD-NCED4) vector and transformed into E. coli. The positive single colony was selected and sequenced.

2.6 Analysis of the specific expression of NCEDs members in licorice

The root treated with PEG at concentrations of $0 \%, 5 \%, 10 \%$ and $15 \%$ were used for RNA extraction. After reverse transcription was completed, real-time PCR was performed. The Real Time PCR conditions were as follows: pre-denaturation at $95^{\circ} \mathrm{C}$ for $3 \mathrm{~min}$, denaturation at $95^{\circ} \mathrm{C}$ for $10 \mathrm{~s}$, annealing at $57{ }^{\circ} \mathrm{C}$ for 30 $\mathrm{s}$, extension at $72{ }^{\circ} \mathrm{C}$ for $30 \mathrm{~s}, 40$ cycles. The primer sequence was shown in Supplementary Materials Table 6. Each experiment was repeated three times.

\subsection{SNPs analysis of NCEDs genes of licorice from different provenances}

The DNA of licorice was extracted from leaves of licorice. According to the full-length sequence of NCED1, NCED3 and NCED4, primers were designed to amplify the three genes in licorice. The PCR reaction system was $30 \mu \mathrm{L}$. the reaction conditions were: $94^{\circ} \mathrm{C}$ pre denaturation for $5 \mathrm{~min}, 94^{\circ} \mathrm{C}$ denaturation for $30 \mathrm{~s}$, annealing at $55^{\circ} \mathrm{C}$ for $1 \mathrm{~min}, 72^{\circ} \mathrm{C}$ extension for $2 \mathrm{~min}, 40$ cycles, $72^{\circ} \mathrm{C}$ extension for $10 \mathrm{~min}$.

\subsection{Overexpression of NCEDs gene}

Tobacco TobRB7 promoter was designed and synthesized according to NCBI sequence. Two restriction sites of Sal I and Bgl II were selected to linearize the vector pCAMBIA1305.1, Sal I and Bgl II double- 
digested TobRB7, and the T4 ligase method was used to ligate it into the vector to generate pCAMBIA1305.1- TobRB7. The pCAMBIA1305.1- TobRB7 was linearized by Bgl II and Spe I restriction enzymes. The NCED1, NCED3, and NCED4 were cloned by Bgl II and Spe I restriction enzymes and the recombinant plasmid was obtained. The recombinant plasmid was transformed into $E$. coli. The cloned recombinant plasmid was extracted with high-purity plasmid small-scale rapid extraction kit (Biomed, Beijing), and transformed into Agrobacterium tumefaciens EHA105 by electric shock transformation. Synthesize EHA-TobRB7-NCED1, EHA-TobRB7-NCED3, EHA-TobRB7-NCED4 engineering bacteria were synthesized. A single colony of engineered bacteria was inoculated into YEB liquid medium and cultured at $28^{\circ} \mathrm{C}$ and $280 \mathrm{rpm}$. The seeds of licorice were soaked in the bacterial solution, cultured for 24 hours and then changed to sterile water for culture and stained with GUS (GUS staining blue proved that the engineered bacteria successfully infects licorice, and the target gene was expressed transiently). The seedlings were infected with EHA-TobRB7-NCED1, EHA-TobRB7-NCED3, EHA-TobRB7-NCED4. After the first dection on GUS, the roots were removed to test the content of GA, ABA and $\beta$-AS content.

\subsection{Data analysis}

All experiments were repeated three times. GA content and ABA content were expressed as mean \pm standard deviation, SPSS22.0 software was used for analysis and ANOVA was used. $P<0.05$ was considered to have significant difference. $P<0.01$ was considered to have extremly significant difference. MATLAB7.0 software was used to analyze the relationship between SNPs of NCEDs gene and GA content. The values of $G, A, T$ and $C$ were assigned to $1,2,3$, and 4 respectively to ensure the elimination of dimensions and the accuracy of modeling.

\section{Results}

3.1 The effect of ABA on the expression of key functional gene $\beta-A S$

As figure 1 shown, at $3 \mathrm{~h}$, the expression of $\beta$-AS of licorice treated with different concentrations of $A B A$ increased with the increase of the treatment concentration. Compared with the control group, there were significant differences in each group. At $6 \mathrm{~h}$, the change trend was similar to that at $3 \mathrm{~h}$. After $12 \mathrm{~h}$, when the ABA concentration was $100 \mathrm{mg} / \mathrm{L}$ or $200 \mathrm{mg} / \mathrm{L}$, the expression of $\beta$-AS decreased. When the concentration was $25 \mathrm{mg} / \mathrm{L}$, the longer the treatment time, the expression of $\beta$-AS showed a downward trend. But there was still a significant difference from the control group. At a concentration of $50 \mathrm{mg} / \mathrm{L}$, the expression of $\beta$-AS showed an upward trend, and there was a significant difference from the control group. All these indicate that treatment of licorice plant seedlings with low concentration of ABA (25-50 $\mathrm{mg} / \mathrm{L})$ can promote the expression of $\beta$-AS, while high-concentration $(100-200 \mathrm{mg} / \mathrm{L})$ treatment will show a downward trend as the treatment time continues.

3.2 Analysis of the GA content and ABA variation of licorice in different provenances

The content of GA was different among different provenances. $Y X$ has the highest GA content. There was no significant difference in GA content between AH and CF and YX. MQ has the lowest GA content (Fig. 
2A). There were also differences in ABA content among different provenances. The ABA content of HJ2 was the highest, which has no significant difference with GD. HT contained the lowest ABA (Fig. 2B). The differences in $G A$ and $A B A$ content within each provenance provide favorable materials for further study of the variation of $G A$ and $A B A$ content.

\subsection{Cloning and functional verification of NCEDs}

The full length of the open reading frames of NCED1, NCED3, and NCED4 were $1830 \mathrm{bp}, 1830 \mathrm{bp}$ and $1764 \mathrm{bp}$, respectively. All three genes had one exon and no intron (supplementary material). The three NCED genes were compared and found to have high similarities with the NCED gene sequences of various plants (Figure 3A, 3B, 3C; supplementary materials). They can encode 609, 609 and 587 amino acids, respectively. The molecular weights of the hypothetical proteins were $67.56 \mathrm{kDa}, 67.29 \mathrm{kDa}$ and $63.98 \mathrm{kDa}$, respectively, and their isoelectric points were $6.64,5.95$, and 6.26 , respectively. After treating licorice with different concentrations of PEG to simulate the effect of drought on the ABA content of licorice and the relative expression of NCEDs gene family members (Figure 3D), the results showed that the ABA content increased with the increase of PEG concentration, $10 \%$ and $15 \%$ had significantly differences compared with the control group. That is, drought stress could promote the increase of ABA content of licorice. As the concentration of PEG increased, the relative expression levels of the three genes all showed an increasing trend (Figure $3 \mathrm{E}$ ). That is, drought stress can significantly increase the relative expression of NCEDs gene family members. At the same time, the expression of NCED3 gene was significantly higher than the other two genes when treated with high concentration, indicating that this gene has a stronger effect under severe drought conditions.

\subsection{SNPs analysis of NCEDs genes of licorice from different provenances}

Among the 13 provenance samples, the numbers of NCED1, NCED3, and NCED4 samples were successfully cloned and sequenced, respectively, 65,67 , and 36 . The numbers of SNPs were found to be 14,14 and 29 after DNAMAN alignment. According to the SNP locus of the NCED1 gene, the samples from 13 provenances were divided into 22 genotypes (Supplementary Materials Table 7), among which the genotype with the largest number of samples was NCED-G1, with a total of 14 samples; according to the SNP locus of the NCED3 gene(Supplementary Materials Table 8), The 13 samples were divided into 29 genotypes, among which the genotype with the largest number of samples was NCED-G23, with 26 samples in total; 13 provenance samples were divided into 27 genotypes according to the SNP locus of the NCED4 gene (Supplementary Materials Table 9). Among them, the genotype with the largest number of samples is NCED-G1, with a total of 9 samples. There are more genotypes for 3 genes and 1 provenance. Since some provenances were distributed in different genotype lengths, it showed that there were rich variations within the provenances, while some genotypes contain samples from different provenances, which showed that there was consistency between different provenances.

3.5 Correlation analysis between SNPs of NCEDs and GA content 
Among all SNPs of NCED1, NCED3 and NCED4, mutation sites with a correlation coefficient greater than 0.8 for GA content accounted for $78.57 \%, 76.92 \%$, and $72.14 \%$ of the corresponding gene mutation sites (Supplementary Materials $13,14,15$ ), indicating three The mutant gene of the patient has a strong promoting effect on the synthesis and accumulation of GA, and the mutation site of NCED1 gene has a better effect on the synthesis and accumulation of ABA during the sampling period than the other two genes. The highest correlation between NCED1 gene and GA content is the SNP site (G/A) at 437 bp with a correlation coefficient of 0.8779 (Supplementary Material Table 10). Its $\mathrm{G}$ type is more conducive to the synthesis and accumulation of GA. NCED3 gene and NCED1 have similar results (Supplementary Materials Table 11). Its $\mathrm{G}$ type is more conducive to the synthesis and accumulation of GA. The highest correlation between NCED4 gene and GA content is the SNP locus at $845 \mathrm{bp}(\mathrm{A} / \mathrm{G})$ with a correlation coefficient of 0.8762 (Supplementary Table 12). Its type $A$ is more conducive to the synthesis and accumulation of GA. These results indicate that the above three loci are the loci that have the greatest impact on GA synthesis and accumulation among the three genes.

\subsection{Revalidation of NCEDs}

After treatment with the engineered bacteria, the expression of $\beta$-ASgene showed a trend of increase (Figure 4A). There were significant differences in the expression of $\beta$-AS gene between the licorice infected by different engineered bacteria and the licorice of the control group. The degree of improvement was EHA- TobRB7-NCED1> EHA- TobRB7-NCED3> EHA- TobRB7-NCED4. Therefore, the G-type at $437 \mathrm{bp}$ of $N C E D 1$ gene sequence had a stronger effect on the increase of $\beta$-AS gene expression during this sampling period than NCED3 and NCED4, but these two also had a certain effect on the accumulation of $\beta$-AS gene expression. In addition, the results of the ABA and GA content of the seedlings treated with the engineered bacteria showed that NCED1 promoted the ABA and GA content better than the other two genes (Figure $4 B, 4 C$ ). In short, the $G$ type at 437 bp of NCED1 gene sequence has a stronger influence on the synthesis and accumulation of GA and ABA during the sampling period than NCED3 and NCED4, but the two also have a certain influence on the synthesis and accumulation of GA and ABA [26].

\section{Discussion}

$A B A$ is an important connection between the plant's response to adversity and secondary metabolites, and the secondary metabolites produced can protect plants from environmental stress, so it can promote the synthesis of certain secondary metabolites. When seedlings were treated with different concentrations of $A B A$, it was found that low concentrations $(25-50 \mathrm{mg} / \mathrm{L})$ of $A B A$ could continuously promote the increase of the relative expression of $\beta$-AS during the synthesis of GA. Therefore, low concentration promoted the increase of GA content by promoting the expression of key genes in the process of GA synthesis.

After planting licorice from wild provenances in 13 major production areas for one and a half years, the GA content of licorice was measured. It was found that significant differences existed not only among the provenances, but also within the provenances. These were mainly determined by the the complex genetic 
background of licorice, which was consistent with previous studies [27]. Phytohormones play an extremely important role in the growth and development of plants, which are mainly manifested by signal transduction to enable plants to adapt to external stimuli and promote their own development [16][28]. The main reason for the insignificant differences in ABA between different provenances was the sampling period, but the larger differences within the same provenance were consistent with the predecessors, which also showed that the genetic background of licorice was more complicated.

NCEDs are one of the key functional genes in the process of ABA synthesis, which play a major role in the process of ABA synthesis. Three members of the NCEDs gene family were cloned from licorice by using a combination of homologous cloning and RACE methods. Bioinformatics analysis found that these three genes had a homology of more than $70 \%$ with the corresponding genes of other plants in the same family. After simulated drought stress, the relative expression of the three genes increased in synchrony with the increase in $A B A$ and $\beta-A S$.

Among the 43 genotypes NCED1, NCED3, and NCED4, the highest correlation coefficients with GA were at 437 bp in NCED1 gene, 966 bp in NCED3 gene, 845 bp in NCED4 gene, and the mutation sites with highest correlation coefficient with ABA were 755 bp in NCED1 gene, 443 bp in NCED3 gene, 1645 bp in NCED4 gene, and the order of the correlation coefficient between GA and the 3 genes with different mutation sites was not consistent with the order of the correlation coefficient between ABA and the 3 genes with different mutation sites. The possible reason was that the appropriate concentration of $A B A$ can promote the synthesis and accumulation of GA, while the high concentration of ABA inhibits the synthesis and accumulation of GA according to our results.

Due to the existence of degenerate codons, the mutation of functional gene SNP has two results: synonymous mutation and missense mutation. However, these two results play a certain role in promoting the synthesis and accumulation of GA and $A B A$ in the association analysis. Missense mutations can affect the catalytic efficiency of proteins to a certain extent by changing the types of amino acids. For example, in NCED3, the correlation coefficient between 4 missense mutation sites and GA content in 6 missense mutation sites was greater than 0.8 \and was located in the first 4 positions of all mutation coefficients. It showed that the missense site was the reason for changing the catalytic efficiency of the enzyme. But there were also cases where missense mutations have less effect, while synonymous mutations had a greater effect. Synonymous mutations may change the helical structure of the DNA sequence through the difference in the charge number of different bases, thus promoting the synthesis and accumulation of certain substances [29].

The biosynthetic pathway of glycyrrhizic acid does not exist in isolation. It is connected with other secondary metabolites such as ABA, GA, glycyrrhizin, glycyrrhizin and other biosynthetic pathways to form a network. NCEDs genes are one of the rate-limiting enzymes in the ABA synthesis pathway, and its expression directly affects the synthesis of $A B A$ and then the activity of glycyrrhizic acid biosynthesis enzymes, regulating the synthesis of GA. 
This study showed that appropriate concentration of $A B A$ treatment can increase the expression of key functional gene $\beta-A S$ in the process of GA synthesis. The NCEDs family members NCED1, NCED3 and NCED4, the key functional genes of ABA, could promote the synthesis of ABA. We collected 115 samples from 13 wild provenances of licorice from different provenances, analyzed their $G A$ and $A B A$ content, and found that the GA and ABA content of different provenances and different individual plants had large variations. More importantly, after the grey correlation analysis between the SNPs of the three genes and the content of ABA and GA, the three NCED family genes were re-verified. The NCED1 gene variant sites were compared to the total synthesis and accumulation of $A B A$ and $G A$ during the sampling period. The role of NCED1 is better than that of NCED3 and NCED4, but the two genes of NCED3 and NCED4 also have a certain effect on the synthesis and accumulation of ABA and GA, that is, NCED1 gene 437 bp G type > NCED3 gene 966 bp G type > NCED4 gene 845 bp A type. Therefore, in the selection of high glycyrrhizic acid germplasm, priority should be given to the $\mathrm{G}$ type at $437 \mathrm{bp}$ in NCED1 gene, $\mathrm{G}$ type at 966 bp in NCED3 gene and A type at 845 bp in NCED4 gene.

These studies have created a new idea: to explain the mechanism of the variation of the regulated end product from the variation of the key regulatory substances in the regulatory network to explain the reasons for the variation of the GA content of different genotypes, and to provide a more direct standard for the selection of high-quality licorice germplasm.

\section{Abbreviations}

GA: Glycyrrhizic Acid

ABA: Abscisic Acid

SNP: Single Nucleotide Polymorphism

NCED: 9-Cis-Epoxycarotenoids Dioxygenase

$\beta$-AS: $\beta$-Aromatic Alcohol Synthase

\section{Declarations}

\section{Acknowledgements}

None.

\section{Authors' contributions}

$T L, F X, D J$ and XS participated in the design of the study $\llbracket$ data analysis and prepared the manuscript. GR and $\mathrm{XY}$ conducted the experiments. $\mathrm{CL}$ is responsible for the overall supervision of the project. All authors read and approve the final manuscript. 


\section{Funding}

This work was supported by the National Natural Science Foundation of China(No.81703645 81773838) Availability of data and materials

The data used and/or investigated during the present study are available from the corresponding author upon reasonable request.

\section{Declarations}

\section{Ethics approval and consent to participate}

No applicable.

\section{Consent for publication}

Not applicable.

\section{Competing interests}

The authors declare that they have no competing interests.

\section{Author details}

${ }^{1}$ School of Chinese Pharmacy, Beijing University of Chinese Medicine, Beijing 102488, China

\section{References}

[1]Yin, Y., Li, Y.P., Jiang, D., Zhang, X.N., Gao, W., and Liu, C.S. (2020). De novo biosynthesis of liquiritin in Saccharomyces cerevisiae. Acta Pharm Sin B 10, 711-721.

[2]Asl, M.N., and Hosseinzadeh, H. (2008). Review of pharmacological effects of Glycyrrhiza sp and its bioactive compounds. Phytother Res 22, 709-724.

[3]Huang, Y., Li, D., Wang, J.H., Cai, Y., Dai, Z.B., Jiang, D., and Liu, C.S. (2019). GuUGT, a glycosyltransferase from Glycyrrhiza uralensis, exhibits glycyrrhetinic acid 3-and 30-0-glycosylation. R Soc Open Sci 6, 9.

[4]Ji, S., Wang, Y.J., Shao, X., Zhu, C.C., Lin, Y., Gao, S.K., and Tang, D.Q. (2020). Extraction and purification of triterpenoid saponins from licorice by ionic liquid based extraction combined with in situ alkaline aqueous biphasic systems. Sep Purif Technol 247, 8.

[5]Kao, T.C., Wu, C.H., and Yen, G.C. (2014). Bioactivity and Potential Health Benefits of Licorice. J Agric Food Chem 62, 542-553. 
[6]Lim, J.W., Seo, J.K., Jung, S.J., and Kang, S.Y.(2021). Efficacy of an optimized extract from licorice roots (Glycyrrhiza uralensis fischer) against viral hemorrhagic septicemia virus in olive flounder (Paralichthys olivaceus). Aquac Res, 52.2609-2621.

[7]Han, Y.-X., Jia, Q.-J., Yang, D.-F., Chai, W.-G., Zhang, X.-M., He, Q.-L., and Liang, Z.-S. (2021). Current advances in environmental stimuli regulating the glycyrrhizic acid biosynthesis pathway. Fitoterapia 151, 104860-104860.

[8]Li, Y.P., Yu, C.X., Qiao, J., Zang, Y.M., Xiang, Y., Ren, G.X., Wang, L., Zhang, X.Y., and Liu, C.S. (2016). Effect of exogenous phytohormones treatment on glycyrrhizic acid accumulation and preliminary exploration of the chemical control network based on glycyrrhizic acid in root of Glycyrrhiza uralensis. Rev Bras Farmacogn-Braz J Pharmacogn 26, 490-496.

[9]Ma, S.J., Zhu, G.W., Yu, F.L., Zhu, G.H., Wang, D., Wang, W.Q., and Hou, J.L. (2018). Effects of manganese on accumulation of Glycyrrhizic acid based on material ingredients distribution of Glycyrrhiza uralensis. Ind Crop Prod 112, 151-159.

[10]Wang, C.C., Chen, L.H., Cai, Z.C., Chen, C.X., Liu, Z.X., Liu, X.H., Zou, L.S., Chen, J.L., Tan, M.X., Wei, L.F., et al. (2019). Dynamic Variations in Multiple Bioactive Constituents under Salt Stress Provide Insight into Quality Formation of Licorice. Molecules 24, 14.

[11]Hou, J.L., Li, W.D., Zheng, Q.Y., Wang, W.Q., Xiao, B., and Xing, D. (2010). Effect of low light intensity on growth and accumulation of secondary metabolites in roots of Glycyrrhiza uralensis Fisch. Biochem Syst Ecol 38, 160-168.

[12]Yu, F.L., Wang, Q.L., Wei, S.L., Wang, D., Fang, Y.Q., Liu, F.B., Zhao, Z.G., Hou, J.L., and Wang, W.Q. (2015). Effect of Genotype and Environment on Five Bioactive Components of Cultivated Licorice (Glycyrrhiza uralensis) Populations in Northern China. Biol Pharm Bull 38, 75-81.

[13]Kojoma, M., Hayashi, S., Shibata, T., Yamamoto, Y., and Sekizaki, H. (2011). Variation of Glycyrrhizin and Liquiritin Contents within a Population of 5-Year-Old Licorice (Glycyrrhiza uralensis) Plants Cultivated under the Same Conditions. Biol Pharm Bull 34, 1334-1337.

[14]Li, L., Zhu, T., Song, Y., Feng, L., Farag, E.A.H., and Ren, M. (2021). ABSCISIC ACID INSENSITIVE5 Interacts With RIBOSOMAL S6 KINASE2 to Mediate ABA Responses During Seedling Growth in Arabidopsis. Frontiers in Plant Science 11.

[15]Pulido, P., Perello, C., and Rodriguez-Concepcion, M. (2012). New Insights into Plant Isoprenoid Metabolism. Mol Plant 5, 964-967.

[16]Shi, X.P., Ren, J.J., Qi, H.D., Lin, Y., Wang, Y.Y., Li, D.F., Kong, L..J., and Wang, X.L. (2021). Plant-Specific AtS40.4 Acts as a Negative Regulator in Abscisic Acid Signaling During Seed Germination and Seedling Growth in Arabidopsis. Frontiers in plant science 12, 622201-622201. 
[17]Zhang, R., Wang, Y., Li, S.H., Yang, L., and Liang, Z.C. (2021). ABA signaling pathway genes and function during abiotic stress and berry ripening in Vitis vinifera. Gene 769, 11.

[18]Hwang, S.G., Lee, C.Y., and Tseng, C.S. (2018). Heterologous expression of rice 9-cis-epoxycarotenoid dioxygenase 4 (OsNCED4) in Arabidopsis confers sugar oversensitivity and drought tolerance. Bot Stud $59,12$.

[19]Bai, Q., Huang, Y., and Shen, Y.Y. (2021). The Physiological and Molecular Mechanism of Abscisic Acid in Regulation of Fleshy Fruit Ripening. Frontiers in Plant Science 11, 9.

[20]Martinez-Andujar, C., Ordiz, M.I., Huang, Z.L., Nonogaki, M., Beachy, R.N., and Nonogaki, H. (2011). Induction of 9-cis-epoxycarotenoid dioxygenase in Arabidopsis thaliana seeds enhances seed dormancy. Proc Natl Acad Sci U S A 108, 17225-17229.

[21]Zakari, S.A., Asad, M.A.U., Han, Z.Y., Zhao, Q., and Cheng, F.M. (2020). Relationship of Nitrogen Deficiency-Induced Leaf Senescence with ROS Generation and ABA Concentration in Rice Flag Leaves. J Plant Growth Regul 39, 1503-1517.

[22]Hu, T., Liu, Z.Z., Tian, S.K., Hou, J.M., Ma, Y.S., Li, W.D., and Liu, Y. (2019). Polymorphism of betaamyrin synthase gene (beta-AS) influence the accumulation of triterpenes in licorice. S Afr J Bot 125, 310320.

[23]Liu, Y., Zhan, X.J., Li, W.D., Gao, Y., Wen, H., Chen, H.H., Wang, L.Q., and Liu, C.S. (2014). Copy number variations of functional genes influence contents of glycyrrhizic acid in Glycyrrhiza uralensis. Acta Physiol Plant 36, 1433-1440.

[24]Durek, P., and Walther, D. (2008). The integrated analysis of metabolic and protein interaction networks reveals novel molecular organizing principles. Bmc Systems Biology 2.

[25]Qiao, J.,Luo Z.L., Li,Y.P., Ren,G.X., Liu,C.S. (2017). Effect of Abscisic Acid on Accumulation of Five Active Components in Root of Glycyrrhiza uralensis. Molecules 22(11): 11.

[26]Yang X.H.. (2018). Study on the catalytic efficiency and prokaryotic expression of different copies of licorice NCEDs,. Beijing University of Traditional Chinese Medicine.

[27]Liu, Y.L., Zhang, P.F., Song, M.L., Hou, J.L., Qing, M., Wang, W.Q., and Liu, C.S. (2015). Transcriptome Analysis and Development of SSR Molecular Markers in Glycyrrhiza uralensis Fisch. PLoS One 10, 12.

[28]Xu, J.X., Li, Q.Z., Li, Y., Yang, L.Y., Zhang, Y.C., and Cai, Y.M. (2021). Effect of Exogenous Gibberellin, Paclobutrazol, Abscisic Acid, and Ethrel Application on Bulblet Development in Lycoris radiata. Frontiers in Plant Science 11, 16.

[29]Ren G.X. (2016).The effect of mutations in ABA key functional genes NCEDs in the glycyrrhizic acid synthesis regulatory network on glycyrrhizic acid synthesis [D]. Beijing University of Traditional Chinese 
Medicine.

Figures

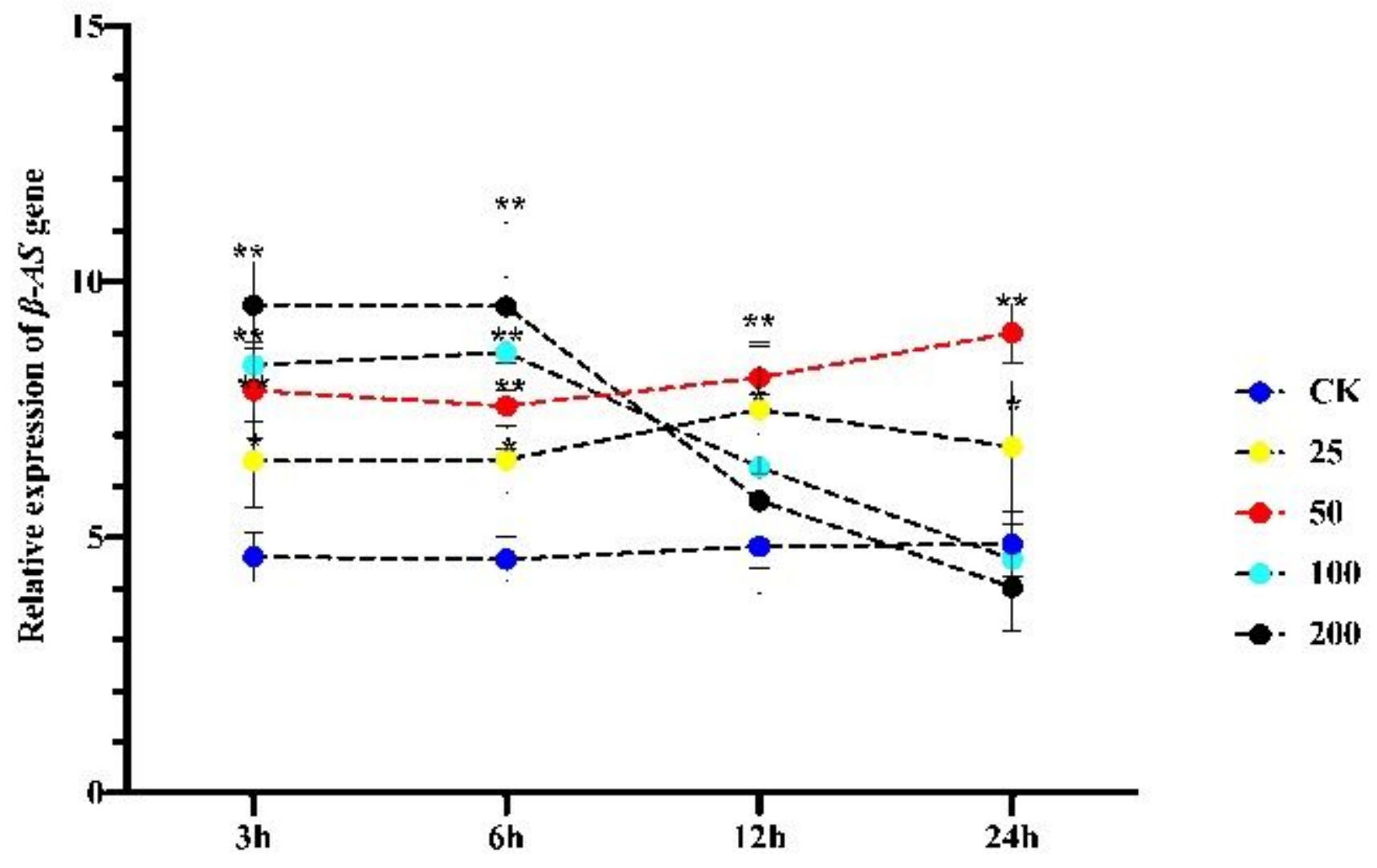

I'ime(h)

Figure 1

Effects of applying different concentrations of ABA on the relative expression of key functional gene $\beta$-AS

A

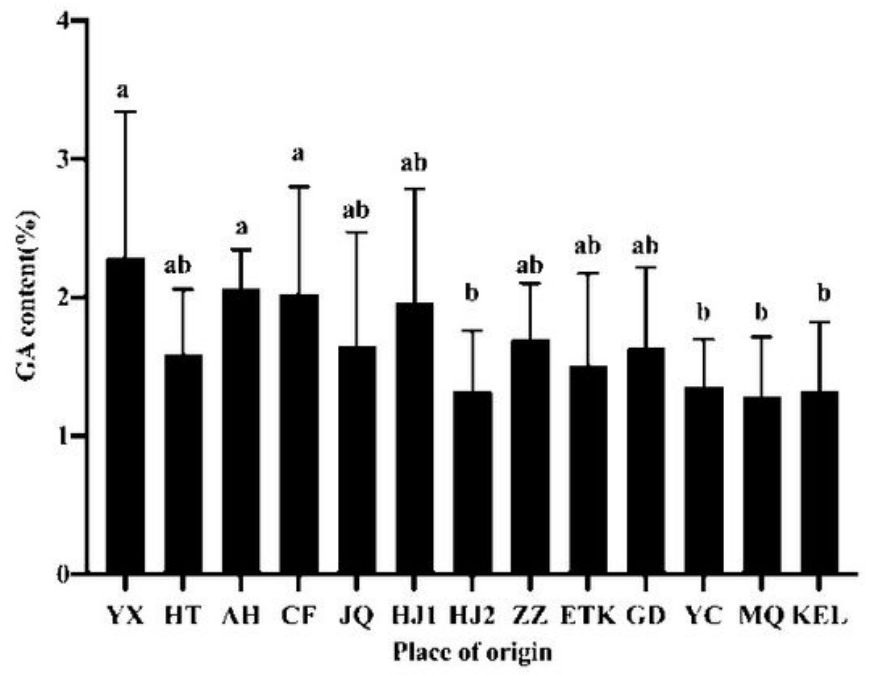

B

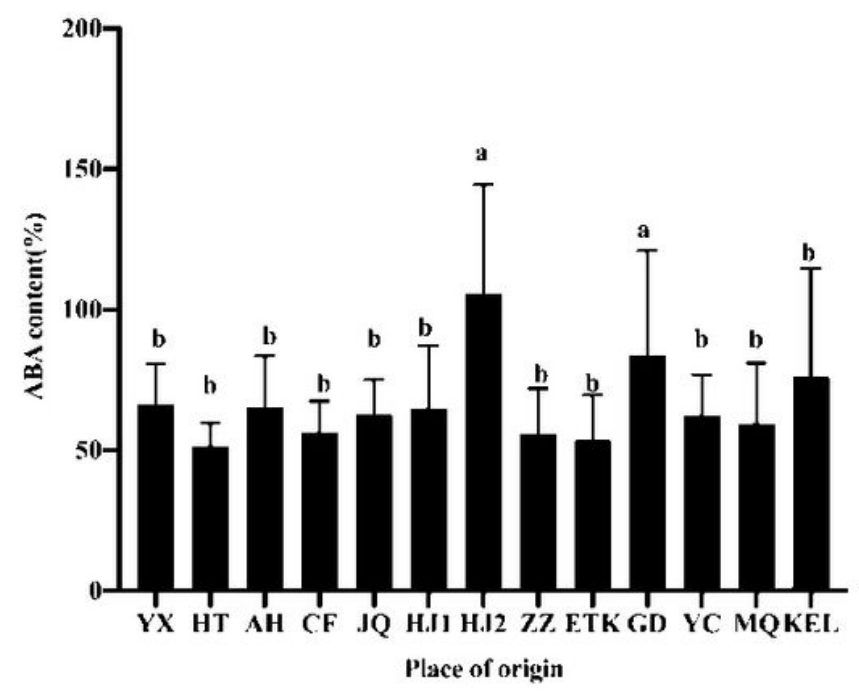


Figure 2

The content of GA and ABA of different provenances (A: The content of GA of different provenances; $B$ : The content of ABA of different provenances)

A

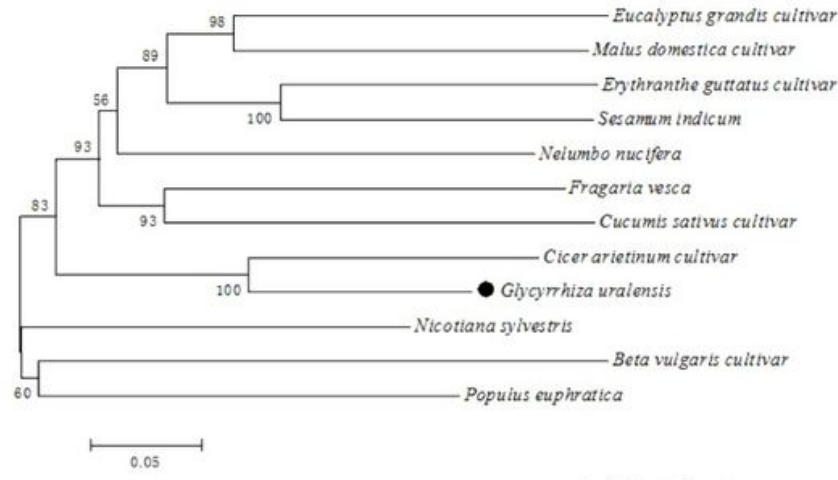

C

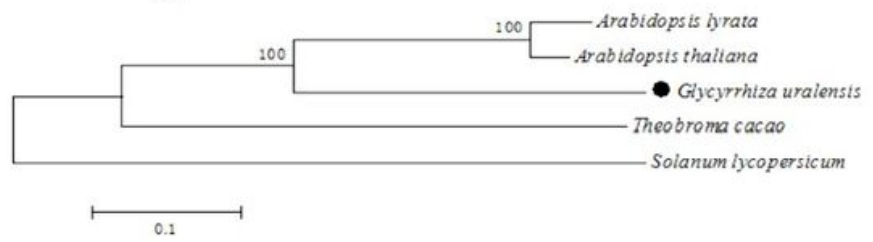

B

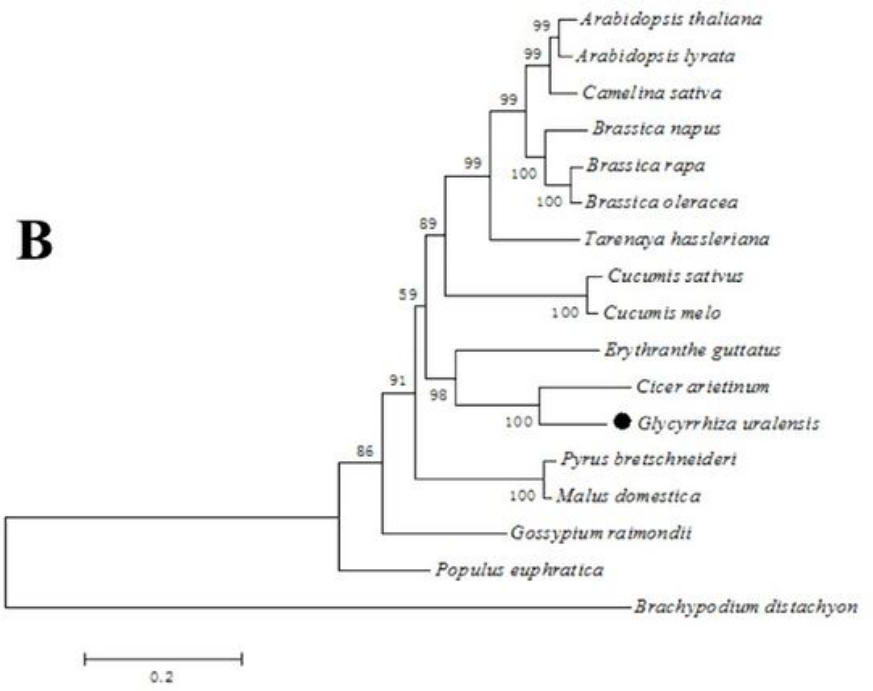

D

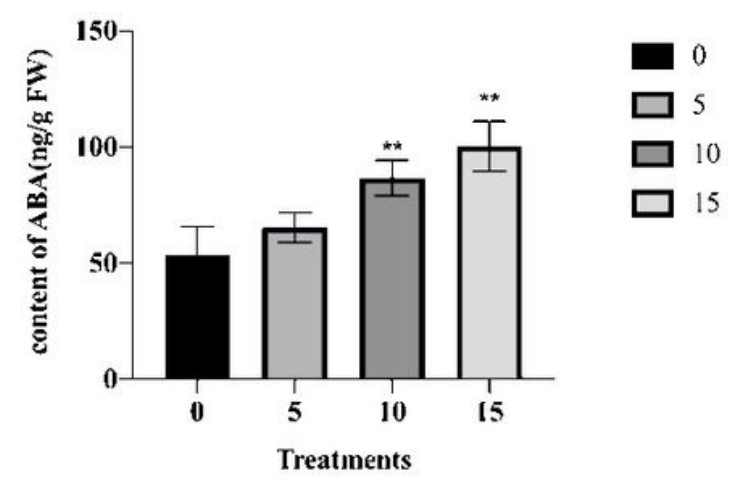

$\mathbf{E}$

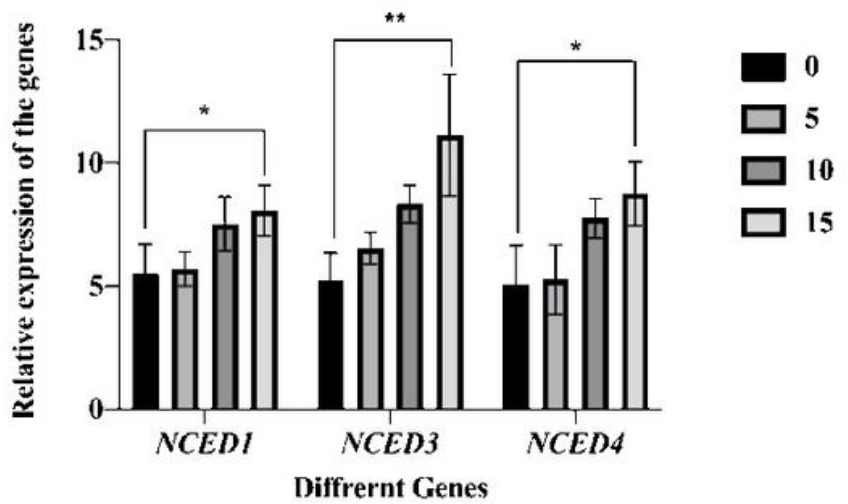

\section{Figure 3}

Phylogenetic tree of NCED s gene of licorice and preliminary verification of function (A: NCED1 has the highest sequence homology with Cicer arietinum Linn; B: NCED3 and Cicer arietinum Linn.; C: NCED4 has the highest sequence homology with Arabidopsis lyrata; $D$ : The effect of different concentrations of PEG treatment on the ABA content of licorice; $E$ : The effect of different concentrations of PEG on the expression of NCEDs family members.) 

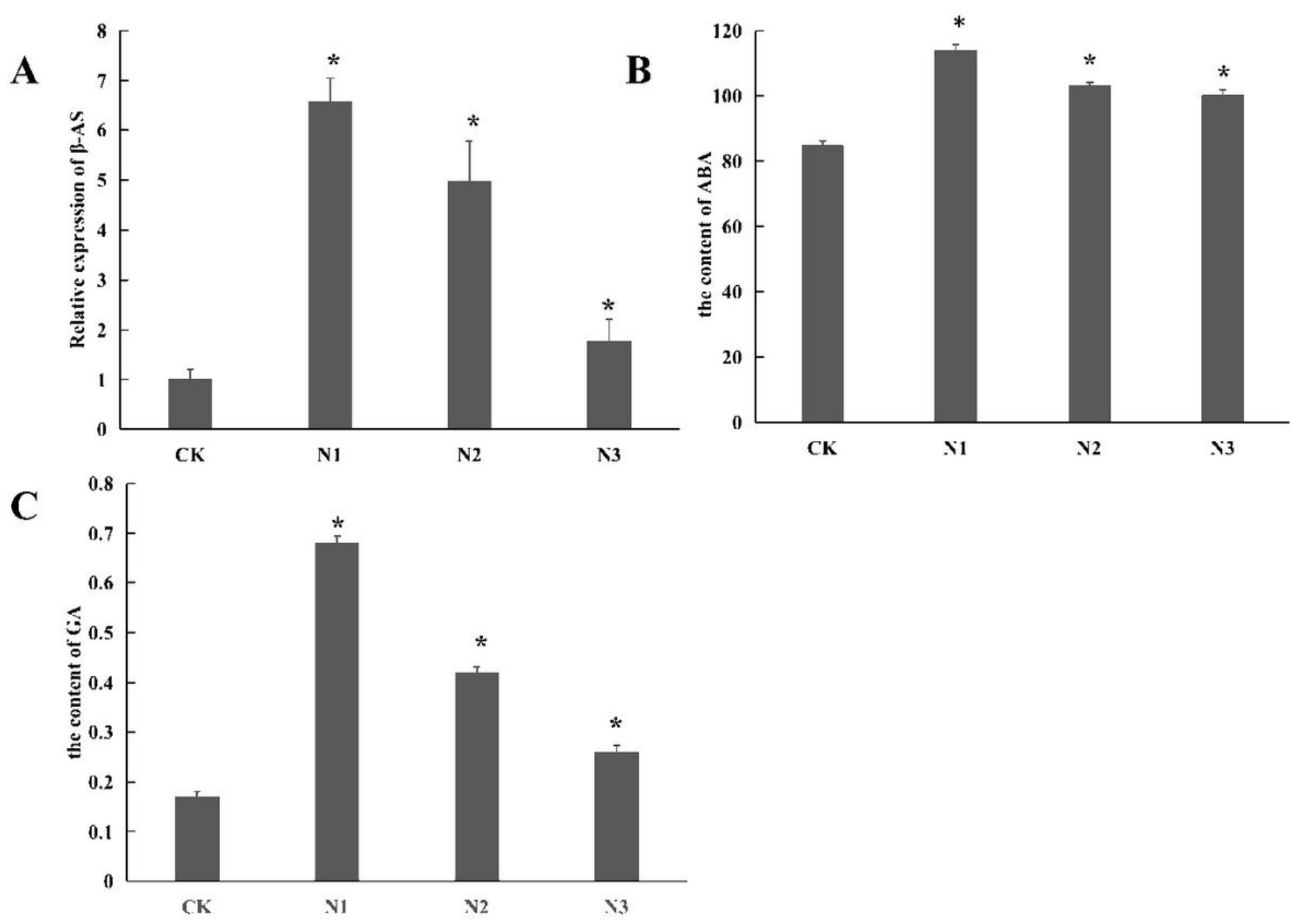

\section{Figure 4}

The effect of NCEDs overexpression on the content of key functional genes $\beta-A S, A B A$ and GA in licorice (A: changes in $\beta$-AS expression; $B$ : changes in ABA content; $C$ : changes in GA content. CK: control group; N1: EHA- TobRB7-NCED1; N3: EHA- TobRB7-NCED3; N4: EHA- TobRB7-NCED4)

\section{Supplementary Files}

This is a list of supplementary files associated with this preprint. Click to download.

- supplementarymaterial.pdf 